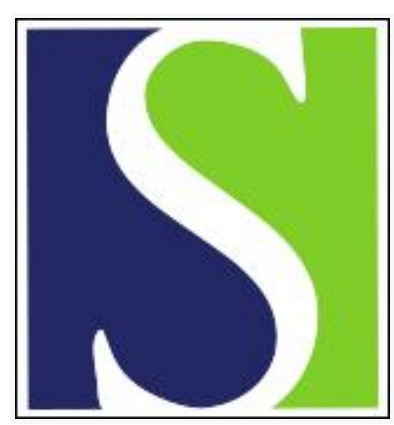

Scand J Work Environ Health 2003;29(3):197-205

https://doi.org/10.5271/sjweh.722

Issue date: Jun 2003

Development of neck and hand-wrist symptoms in relation to duration of computer use at work

by Jensen $C$

Affiliation: National Institute of Occupational Health, Lersø Parkalle, DK-2100 Copenhagen, Denmark. cj@ami.dk

Refers to the following texts of the Journal: 1998;24(5):418-424

2000;26(5):390-397

The following articles refer to this text: 2004;30(5):390-398;

2004;30(5):399-409; SJWEH Supplements 2007;(3):26-32;

2008;34(1):55-65; 2008;34(2):120-132; 2009;35(2):134-144

Key terms: computer use; duration of computer use; follow-up study; hand; hand-wrist symptom; mouse use; neck; neck symptom; psychosocial factor; work; wrist

This article in PubMed: www.ncbi.nlm.nih.gov/pubmed/12828389

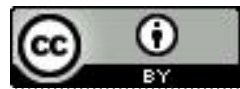




\title{
Development of neck and hand-wrist symptoms in relation to duration of computer use at work
}

\author{
by Chris Jensen, $P h D^{1}$
}

\begin{abstract}
Jensen C. Development of neck and hand-wrist symptoms in relation to duration of computer use at work. Scand J Work Environ Health2003;29(3):197-205.
\end{abstract}

Objectives This study attempted to identify risk factors for musculoskeletal symptoms in the neck and handwrist regions among employees using computers at work.

Methods Computer users ( $N=5033)$ first received a questionnaire in the beginning of 1999 (69\% response rate, $\mathrm{N}=3475$ ), and a follow-up questionnaire was mailed in December of 2000 to the 3361 respondents to the baseline survey ( $77 \%$ response rate, $\mathrm{N}=2576$ ). Health outcome was defined as musculoskeletal symptoms for $>7$ days within the last year of follow-up among the nonsymptomatic respondents at baseline.

Results Men's and women's previous symptoms, women's low influence at work and high-placed computer screen, and men's short time in the same job and good computer skills were associated with neck symptoms. Hand-wrist symptoms were predicted by previous symptoms and low influence at work for both the men and women and sensorial demands for the women only. The duration of computer use predicted hand-wrist symptoms [eg, odds ratio (OR) of 2.3, 95\% confidence interval (95\% CI) 1.2-4.3, for almost continual computer use], but not neck symptoms. For those with almost continual computer use, hand-wrist symptoms were associated with mouse use for at least half of the worktime (OR 4.0, 95\% CI 1.0-15.5) and not using the mouse at all (OR 4.0, 95\% CI 1.1-14.4), as compared with mouse use for one-fourth of the worktime.

Conclusions Limiting computer use to less than three-fourths of the worktime would help to prevent handwrist symptoms. Furthermore, low influence at work predicts both neck and hand-wrist symptoms.

Key terms computer use, follow-up study, hand, wrist, mouse use, neck, psychosocial factors.

Neck and upper extremity symptoms have been reported in different types of jobs that involve intense computer use (1). My paper examines risk factors for neck and hand-wrist symptoms among computer users on the basis of a longitudinal study, the BIT study (BIT = behavior in information technology).

In a literature review, Punnett \& Bergqvist (2) reported that poor workstation design, continuous computer use for the entire workday, and repetitive computer work such as data entry were associated with an increased risk of symptoms. Several studies have reported positive associations between duration of computer use and musculoskeletal symptoms (1, 3-7). Karlqvist et al (6) and Jensen et al (8) reported a high prevalence of musculoskeletal symptoms among CAD (computeraided design) operators, who used a computer mouse intensively at work. In addition, other factors in jobs with intensive computer work, such as low job control, overtime work, and frequent deadlines, have been reported to be associated with musculoskeletal symptoms $(5,9,10)$. However, most results were based on crosssectional studies in which cause-response relationships were difficult to identify.

In a recent follow-up study on newly hired employees with computer use for more than 15 hours a week and a follow-up period of 3 years, incident neck-shoulder and hand-arm (including wrist) symptoms were frequent (11). The women were more at risk than the men, and the risk of developing hand-arm symptoms, but not neck-shoulder symptoms, increased with the duration of weekly keyboard use $(11,12)$. However, the effects of the total duration of computer use (including keying,

Reprint requests to: Dr Chris Jensen, National Institute of Occupational Health, Lersø Parkalle 105, DK-2100 Copenhagen, Denmark. [E-mail: cj@ami.dk] 
using the mouse, and reading on the screen) or mouse use were not studied. In addition, in one older followup study with two measurements at a 6-year interval, a positive association was found between weekly hours of computer use and hand-wrist symptoms, but not between weekly hours of compute use and neck-shoulder symptoms (13).

The aim of this follow-up study was to investigate risk factors for musculoskeletal symptoms in the neck and hand-wrist regions with special focus on the duration of computer use at work. The main hypotheses were that long hours of computer and mouse use are risk factors for the development of symptoms. Analyses were also performed to identify other potential risk factors. These were work-related psychosocial factors, physical factors related to workplace design, individual factors, and factors specifically related to computer use, such as computer skills and quality of technical support.

\section{Subjects and methods}

\section{Subjects}

Two questionnaires were delivered to computer users in 11 Danish companies and institutions. The companies were selected to provide employees with different types of computer work (ie, data entry, word processing, graphic work, etc). Thus the employees of the participating companies were computer users with different types of computer work, but the distribution of computer use or worktasks could not be assumed to be strictly representative of office work in general. The baseline questionnaire was delivered to 5033 computer users in the early part of 1999. All employees working in the entire company or in specific predefined departments were included except cleaning and kitchen personnel. Before the questionnaires were delivered to the workplace, the names of the employees were supplied by the company. The questionnaires were returned by mail or returned in sealed envelopes at their workplace and later collected by a representative from the National Institute of Occupational Health.

The response rate at baseline was $69 \%(\mathrm{~N}=3475)$. In the baseline questionnaire people were asked about their home address in order to mail the follow-up questionnaire to as many of the respondents from the baseline survey as possible. The follow-up questionnaire was mailed in December of 2000 to 3471 respondents who had provided their home address. Of those, 108 respondents had changed home address and were not traceable through postal service registers at the time of the follow-up. Thus 3363 respondents were finally assumed to have received the follow-up questionnaire, and 2576 people responded, corresponding to a response rate of $77 \%$. The mean follow-up period was 21 (range 17-23) months.

\section{Questionnaire}

In a previous paper on the baseline survey, a model was described that included individual and work-related factors that could affect the development of musculoskeletal symptoms (1). The baseline questionnaire contained questions on the following factors (all factors, except age, referred specifically to conditions at work): age, duration of employment in same job, years of using a computer, duration of computer use, duration of mouse use, training in computer use, software skills, technical problems with computer, quality of technical support, space for arm support in front of keyboard, screen height, glare, repetitiveness, quantitative demands, sensorial demands, cognitive demands, influence, possibilities for development, and social support. Most of the individual and physical workplace factors were assessed on the basis of answers to one question for each factor. The question on duration of computer use was "How much of your worktime do you work with a computer (including overtime and worktime at home)?", and the answer categories were never-seldom or one-fourth, one-half, three-fourths or almost all of the worktime. The question on duration of mouse use was similar. Continuous variables were categorized in the analyses. Some of the categorical responses were merged into fewer categories to obtain a more balanced distribution of responses. Some, mostly psychosocial factors, were assessed by the construction of indices based on the answer to three to seven different questions each with five response categories (never, seldom, sometimes, often, always). The indices ranged from 0 to 100 for each factor (14). In the further analyses, the index values were categorized as "low" (index value within 0-25 percentile), "medium low" (index value within 25-50 percentile), "medium high" (index value within 50-75 percentile) and "high" (index value within 75-100 percentile).

Both the baseline and follow-up questionnaire contained questions on musculoskeletal symptoms (trouble, ache, or pain) according to a modified version of the Nordic questionnaire (15). Health outcome was defined as (i) neck symptoms (not including shoulder) or (ii) hand-wrist symptoms for more than 7 days within the last year of the follow-up period. Only nonsymptomatic respondents at baseline (no symptoms or symptoms for no more than 7 days within the last year) were included in the analyses. When the development of neck symptoms was analyzed, the respondents were only included if they had been nonsymptomatic at baseline in both the neck and shoulder regions. For the analyses on the development of hand-wrist symptoms, only the 
respondents who had been nonsymptomatic at baseline in both the hand-wrist and elbow regions were included. Thus, while the case definitions were based specifically for the neck and hand-wrist regions, the inclusion of nonsymptomatic respondents at baseline also required that the adjacent regions be symptom-free (shoulder and elbow, respectively). The inclusion of subjects with symptoms for 1-7 days within the last year at baseline among the "nonsymptomatic" respondents allowed for the inclusion of the factor, "previous symptoms", in the analyses of factors that could predict the development of symptoms at follow-up.

\section{Statistics}

Analyses of associations between factors reported at baseline and symptoms at follow-up in the (i) neck and (ii) one or both hands-wrists were performed. Univariate analyses were performed using a significance level of $\mathrm{P}<0.10$. The chi-square or Mantel Haenszel chisquare statistic was used. In multivariate logistic regression analyses, all the significant factors from the univariate analyses were initially included in the model for each body region. Factors were successively omitted from the models if the likelihood ratio test produced probabilities larger than 0.10 . The final logistic regression models were recalculated using only the respondents who did not change jobs between the baseline and follow-up, to minimize potential effects related to a change in exposure during the follow-up period.

The factors "duration of computer use" and "duration of mouse use" were entered into separate logistic regression analyses including only subjects working for 32-41 hours a week (duration of computer and mouse use were assessed as fractions of the worktime) and who did not change jobs between the baseline and followup. The logistic regression model describing the association between symptoms and the duration of computer use was adjusted for gender, age, and all other factors that were significantly associated with symptoms in the previous analyses.

As the duration of mouse use is highly correlated with duration of computer use, the association between mouse use and symptoms was studied in a subgroup of women who worked all or almost all of their worktime at a computer. The men were excluded since there were only a few men working almost all their worktime at the computer. Logistic regression models between duration of mouse use and symptoms were calculated with adjustment for other factors associated with symptoms. The procedures FREQ, MEAN and GENMOD in SAS (version 6.02, Cary, NC, USA) were used. The likelihood ratio test probability was calculated for each factor in the logistic regression models with a significance level of $\mathrm{P}<0.10$.

\section{Results}

In the follow-up, 1721 women and 855 men responded. At baseline the mean age of the female respondents was 42 (SD 10) years, and, on the average, they had used a computer at work for 11 (SD 6) years. The mean age of the men was 43 (SD 10) years, and they had also used a computer at work for 11 (SD 7) years.

At baseline $66.5 \%$ of the 3475 respondents and at follow-up $66.8 \%$ of the 2576 respondents were women. At baseline the prevalence of neck and hand-wrist symptoms for more than 7 days within the last year was 44.7\% ( $\mathrm{N}=2533)$ and $25.8 \%(\mathrm{~N}=2539)$, respectively, when only the subjects who responded at the follow-up were included. At follow-up the prevalence of neck symptoms was $46.4 \%(\mathrm{~N}=2558)$, and the prevalence of hand-wrist symptoms was $31.1 \%(\mathrm{~N}=2514)$. However, at baseline, the prevalences of symptoms of those who responded at follow-up were similar to the prevalences of symptoms for all the participants who responded at baseline (including those who dropped out at follow-up). Thus $43.9 \%$ reported neck symptoms $(\mathrm{N}=3400)$, and $25.9 \%$ reported hand-wrist symptoms $(\mathrm{N}=3405)$ at baseline.

For those who held the same job at follow-up $(\mathrm{N}=2002)$, as compared with those who did not $(\mathrm{N}=458)$, the prevalence of neck symptoms ( $44 \%$ and $46 \%$, respectively) and hand-wrist symptoms (27\% and $22 \%$, respectively) was also similar at baseline. The percentage of the respondents with computer use for the whole worktime was $47 \%$ for those who held the same job at follow-up and $43 \%$ for those who did not.

For the respondents who were nonsymptomatic at baseline, the percentages of incident cases at follow-up are shown in table 1. A greater percentage of the women than the men developed symptoms in both body regions.

Those with previous symptoms (1-7 days within the last year at baseline) were more likely to develop symptoms ( $>7$ days in the follow-up period) than the respondents who reported no symptoms at baseline (table 2). Furthermore, in the univariate analyses, high sensorial demands, low influence at work, high degree of repetitiveness, low social support, and disturbance by glare

Table 1. Incident cases at follow-up. Only respondents with symptoms for $\leq 7$ days during the year preceding the baseline survey have been included.

\begin{tabular}{|c|c|c|c|c|c|c|}
\hline \multirow[t]{2}{*}{ Region } & \multicolumn{3}{|c|}{ Women } & \multicolumn{3}{|c|}{ Men } \\
\hline & $\mathrm{N}$ & $\%$ & $\mathrm{~N}_{\text {total }}{ }^{a}$ & $\mathrm{~N}$ & $\%$ & $\mathrm{~N}_{\text {total }}{ }^{a}$ \\
\hline Neck & 168 & 25.5 & 660 & 82 & 15.4 & 532 \\
\hline Hand-wrist & 223 & 21.6 & 1035 & 78 & 12.5 & 626 \\
\hline
\end{tabular}

${ }^{a} \mathrm{~N}_{\text {total }}=$ total number of nonsymptomatic respondents at baseline. 
Table 2. Percentages of the female and male respondents with symptoms at follow-up according to individual and exposure factors at baseline (percentage of women and men with symptoms $>7$ days during the last year of the follow-up period). P-value refers to chisquare or Mantel Haenszel chi-square test of association.

\begin{tabular}{|c|c|c|c|c|c|c|c|c|c|c|c|c|}
\hline \multirow{3}{*}{ Factor } & \multicolumn{6}{|c|}{ Women } & \multicolumn{6}{|c|}{ Men } \\
\hline & \multicolumn{3}{|c|}{ Neck symptoms } & \multicolumn{3}{|c|}{ Hand-wrist symptoms } & \multicolumn{3}{|c|}{ Neck symptoms } & \multicolumn{3}{|c|}{ Hand-wrist symptoms } \\
\hline & $\%$ & P-value & $\mathrm{N}_{\text {otal }} \mathrm{a}^{\mathrm{a}}$ & $\%$ & P-value & $\mathrm{N}_{\text {total }} \mathrm{a}^{\mathrm{a}}$ & $\%$ & P-value & $\mathrm{N}_{\text {otal }} \mathrm{a}^{\mathrm{a}}$ & $\%$ & P-value & $\mathrm{N}_{\text {total }}{ }^{\mathrm{a}}$ \\
\hline \multicolumn{13}{|l|}{ Age } \\
\hline $\begin{array}{l}18-29 \text { years } \\
30-39 \text { years } \\
40-49 \text { years. } \\
\geq 50 \text { years }\end{array}$ & $\begin{array}{l}28 \\
28 \\
25 \\
23\end{array}$ & $\begin{array}{l}0.28 \\
\cdot \\
\cdot\end{array}$ & $\begin{array}{r}71 \\
163 \\
228 \\
198\end{array}$ & $\begin{array}{l}19 \\
23 \\
23 \\
19\end{array}$ & $\begin{array}{l}0.59 \\
\cdot \\
\cdot\end{array}$ & $\begin{array}{l}113 \\
278 \\
363 \\
281\end{array}$ & $\begin{array}{r}20 \\
21 \\
18 \\
8\end{array}$ & $\begin{array}{l}0.004 \\
\cdot \\
\cdot\end{array}$ & $\begin{array}{r}46 \\
115 \\
193 \\
178\end{array}$ & $\begin{array}{r}7 \\
15 \\
14 \\
11\end{array}$ & $\begin{array}{l}0.99 \\
\cdot \\
\cdot\end{array}$ & $\begin{array}{r}59 \\
147 \\
228 \\
192\end{array}$ \\
\hline \multicolumn{13}{|l|}{ Previous symptoms } \\
\hline $\begin{array}{l}\text { No } \\
\text { Yes, } 1-7 \text { days }\end{array}$ & $\begin{array}{l}15 \\
34\end{array}$ & $<0.001$ & $\begin{array}{l}298 \\
362\end{array}$ & $\begin{array}{l}15 \\
35\end{array}$ & $<0.001$ & $\begin{array}{l}690 \\
345\end{array}$ & $\begin{array}{r}8 \\
24\end{array}$ & $<0.001$ & $\begin{array}{l}295 \\
237\end{array}$ & $\begin{array}{r}8 \\
22\end{array}$ & $<0.001$ & $\begin{array}{l}426 \\
200\end{array}$ \\
\hline \multicolumn{13}{|l|}{ Duration of employment in same job } \\
\hline $\begin{array}{l}<1 \text { year } \\
1-3 \text { years } \\
\geq 4 \text { years }\end{array}$ & $\begin{array}{l}25 \\
32 \\
22\end{array}$ & $\begin{array}{l}0.22 \\
\cdot\end{array}$ & $\begin{array}{l}127 \\
190 \\
331\end{array}$ & $\begin{array}{l}25 \\
21 \\
21\end{array}$ & $\begin{array}{l}0.25 \\
\cdot \\
\cdot\end{array}$ & $\begin{array}{l}194 \\
294 \\
522\end{array}$ & $\begin{array}{l}22 \\
16 \\
13\end{array}$ & $\begin{array}{l}0.034 \\
\cdot\end{array}$ & $\begin{array}{r}91 \\
178 \\
253\end{array}$ & $\begin{array}{l}12 \\
16 \\
11\end{array}$ & $\begin{array}{l}0.38 \\
\cdot\end{array}$ & $\begin{array}{l}107 \\
210 \\
300\end{array}$ \\
\hline \multicolumn{13}{|l|}{ Experience with computer use } \\
\hline $\begin{array}{l}0-3 \text { years } \\
4-7 \text { years } \\
8-12 \text { years } \\
>12 \text { years }\end{array}$ & $\begin{array}{l}31 \\
28 \\
23 \\
25\end{array}$ & $\begin{array}{l}0.26 \\
\cdot \\
\cdot\end{array}$ & $\begin{array}{r}81 \\
98 \\
235 \\
218\end{array}$ & $\begin{array}{l}21 \\
23 \\
23 \\
20\end{array}$ & $\begin{array}{l}0.87 \\
\cdot \\
\cdot\end{array}$ & $\begin{array}{l}132 \\
161 \\
355 \\
345\end{array}$ & $\begin{array}{l}15 \\
11 \\
21 \\
13\end{array}$ & $\begin{array}{l}0.90 \\
\cdot \\
\cdot\end{array}$ & $\begin{array}{r}67 \\
81 \\
180 \\
183\end{array}$ & $\begin{array}{r}9 \\
13 \\
13 \\
12\end{array}$ & $\begin{array}{l}0.60 \\
\cdot \\
\cdot\end{array}$ & $\begin{array}{r}217 \\
208 \\
101 \\
80\end{array}$ \\
\hline \multicolumn{13}{|l|}{ Quantitative demands } \\
\hline $\begin{array}{l}\text { High } \\
\text { Medium high } \\
\text { Medium low } \\
\text { Low }\end{array}$ & $\begin{array}{l}25 \\
29 \\
28 \\
21\end{array}$ & $\begin{array}{l}0.51 \\
\cdot \\
\cdot\end{array}$ & $\begin{array}{l}215 \\
134 \\
140 \\
165\end{array}$ & $\begin{array}{l}23 \\
23 \\
20 \\
18\end{array}$ & $\begin{array}{l}0.12 \\
\cdot \\
\cdot\end{array}$ & $\begin{array}{l}346 \\
243 \\
208 \\
226\end{array}$ & $\begin{array}{l}16 \\
19 \\
13 \\
12\end{array}$ & $\begin{array}{l}0.28 \\
\cdot \\
\cdot\end{array}$ & $\begin{array}{r}242 \\
100 \\
83 \\
104\end{array}$ & $\begin{array}{l}12 \\
11 \\
13 \\
15\end{array}$ & $\begin{array}{l}0.51 \\
\cdot \\
\cdot\end{array}$ & $\begin{array}{l}290 \\
124 \\
100 \\
110\end{array}$ \\
\hline \multicolumn{13}{|l|}{ Cognitive demands } \\
\hline $\begin{array}{l}\text { High } \\
\text { Medium high } \\
\text { Medium low } \\
\text { Low }\end{array}$ & $\begin{array}{l}26 \\
22 \\
32 \\
23\end{array}$ & $\begin{array}{l}0.86 \\
\cdot \\
\cdot\end{array}$ & $\begin{array}{l}176 \\
179 \\
126 \\
172\end{array}$ & $\begin{array}{l}20 \\
21 \\
26 \\
20\end{array}$ & $\begin{array}{l}0.84 \\
\cdot \\
\cdot\end{array}$ & $\begin{array}{l}279 \\
294 \\
200 \\
250\end{array}$ & $\begin{array}{r}17 \\
19 \\
9 \\
11\end{array}$ & $\begin{array}{l}0.094 \\
\cdot \\
\cdot\end{array}$ & $\begin{array}{r}187 \\
161 \\
82 \\
96\end{array}$ & $\begin{array}{l}13 \\
12 \\
10 \\
16\end{array}$ & $\begin{array}{l}0.65 \\
\cdot \\
\cdot\end{array}$ & $\begin{array}{l}225 \\
190 \\
102 \\
105\end{array}$ \\
\hline \multicolumn{13}{|l|}{ Sensory demands } \\
\hline $\begin{array}{l}\text { High } \\
\text { Medium high } \\
\text { Medium low } \\
\text { Low }\end{array}$ & $\begin{array}{l}28 \\
27 \\
19 \\
21\end{array}$ & $\begin{array}{l}0.049 \\
\cdot \\
\cdot\end{array}$ & $\begin{array}{r}259 \\
175 \\
80 \\
140\end{array}$ & $\begin{array}{l}26 \\
18 \\
13 \\
21\end{array}$ & $\begin{array}{l}0.040 \\
\cdot \\
\cdot \\
\cdot\end{array}$ & $\begin{array}{l}415 \\
285 \\
112 \\
215\end{array}$ & $\begin{array}{l}12 \\
21 \\
18 \\
10\end{array}$ & $\begin{array}{l}0.15 \\
\cdot \\
\cdot\end{array}$ & $\begin{array}{r}90 \\
156 \\
95 \\
187\end{array}$ & $\begin{array}{l}11 \\
12 \\
16 \\
12\end{array}$ & $\begin{array}{l}0.68 \\
\cdot \\
\cdot\end{array}$ & $\begin{array}{l}114 \\
185 \\
108 \\
215\end{array}$ \\
\hline \multicolumn{13}{|l|}{ Influence at work } \\
\hline $\begin{array}{l}\text { Low } \\
\text { Medium low } \\
\text { Medium high } \\
\text { High }\end{array}$ & $\begin{array}{l}32 \\
30 \\
23 \\
17\end{array}$ & $\begin{array}{l}<0.001 \\
\cdot\end{array}$ & $\begin{array}{l}170 \\
151 \\
166 \\
166\end{array}$ & $\begin{array}{l}28 \\
25 \\
19 \\
13\end{array}$ & $\begin{array}{l}<0.001 \\
\cdot \\
\cdot\end{array}$ & $\begin{array}{l}287 \\
256 \\
220 \\
257\end{array}$ & $\begin{array}{l}17 \\
16 \\
17 \\
14\end{array}$ & $\begin{array}{l}0.58 \\
\cdot \\
\cdot\end{array}$ & $\begin{array}{r}54 \\
100 \\
127 \\
45\end{array}$ & $\begin{array}{r}12 \\
17 \\
16 \\
8\end{array}$ & $\begin{array}{l}0.050 \\
\cdot \\
\cdot\end{array}$ & $\begin{array}{r}60 \\
122 \\
171 \\
268\end{array}$ \\
\hline \multicolumn{13}{|l|}{ Developmental possibilities } \\
\hline $\begin{array}{l}\text { Low } \\
\text { Medium low } \\
\text { Medium high } \\
\text { High }\end{array}$ & $\begin{array}{l}28 \\
23 \\
27 \\
24\end{array}$ & $\begin{array}{l}0.44 \\
\cdot \\
\cdot\end{array}$ & $\begin{array}{l}150 \\
118 \\
210 \\
176\end{array}$ & $\begin{array}{l}24 \\
19 \\
24 \\
17\end{array}$ & $\begin{array}{l}0.17 \\
\cdot \\
\cdot\end{array}$ & $\begin{array}{l}255 \\
185 \\
342 \\
241\end{array}$ & $\begin{array}{l}10 \\
18 \\
18 \\
14\end{array}$ & $\begin{array}{l}0.74 \\
\cdot \\
\cdot\end{array}$ & $\begin{array}{r}62 \\
72 \\
190 \\
204\end{array}$ & $\begin{array}{l}14 \\
14 \\
12 \\
12\end{array}$ & $\begin{array}{l}0.53 \\
\cdot \\
\cdot\end{array}$ & $\begin{array}{r}72 \\
76 \\
238 \\
237\end{array}$ \\
\hline \multicolumn{13}{|l|}{ Repetitiveness } \\
\hline $\begin{array}{l}\text { Repetitive tasks and movements } \\
\text { Repetitive movements } \\
\text { Varied work }\end{array}$ & $\begin{array}{l}31 \\
25 \\
23\end{array}$ & $\begin{array}{l}0.080 \\
0\end{array}$ & $\begin{array}{l}163 \\
196 \\
300\end{array}$ & $\begin{array}{l}27 \\
21 \\
19\end{array}$ & $\begin{array}{l}0.013 \\
\cdot\end{array}$ & $\begin{array}{l}270 \\
301 \\
456\end{array}$ & $\begin{array}{l}14 \\
18 \\
15\end{array}$ & $\begin{array}{l}0.86 \\
\cdot\end{array}$ & $\begin{array}{r}35 \\
73 \\
421\end{array}$ & $\begin{array}{l}16 \\
14 \\
12\end{array}$ & $\begin{array}{l}0.42 \\
\cdot\end{array}$ & $\begin{array}{r}38 \\
93 \\
491\end{array}$ \\
\hline \multicolumn{13}{|l|}{ Social support } \\
\hline $\begin{array}{l}\text { Low } \\
\text { Medium low } \\
\text { Medium high } \\
\text { High }\end{array}$ & $\begin{array}{l}32 \\
25 \\
24 \\
23\end{array}$ & $\begin{array}{l}0.087 \\
\cdot \\
\cdot\end{array}$ & $\begin{array}{l}114 \\
143 \\
179 \\
217\end{array}$ & $\begin{array}{l}28 \\
21 \\
21 \\
20\end{array}$ & $\begin{array}{l}0.086 \\
\cdot \\
\cdot \\
\cdot\end{array}$ & $\begin{array}{l}173 \\
223 \\
271 \\
355\end{array}$ & $\begin{array}{l}13 \\
12 \\
18 \\
17\end{array}$ & $\begin{array}{l}0.21 \\
\cdot \\
\cdot\end{array}$ & $\begin{array}{l}105 \\
115 \\
146 \\
161\end{array}$ & $\begin{array}{l}10 \\
12 \\
12 \\
15\end{array}$ & $\begin{array}{l}0.21 \\
\cdot \\
\cdot\end{array}$ & $\begin{array}{l}121 \\
149 \\
165 \\
186\end{array}$ \\
\hline \multicolumn{13}{|l|}{ Training in software use } \\
\hline $\begin{array}{l}\text { Insufficient } \\
\text { Sufficient }\end{array}$ & $\begin{array}{l}26 \\
26\end{array}$ & 0.98 & $\begin{array}{l}109 \\
543\end{array}$ & $\begin{array}{l}19 \\
22\end{array}$ & 0.36 & $\begin{array}{l}172 \\
850\end{array}$ & $\begin{array}{l}10 \\
17\end{array}$ & 0.066 & $\begin{array}{l}104 \\
420\end{array}$ & $\begin{array}{l}12 \\
12\end{array}$ & 1.00 & $\begin{array}{l}129 \\
491\end{array}$ \\
\hline \multicolumn{13}{|l|}{ Computer skills } \\
\hline $\begin{array}{l}\text { Bad or somewhat good } \\
\text { Good } \\
\text { Extremely good }\end{array}$ & $\begin{array}{l}27 \\
25 \\
26\end{array}$ & 0.89 & $\begin{array}{r}84 \\
307 \\
261\end{array}$ & $\begin{array}{l}23 \\
22 \\
20\end{array}$ & $\begin{array}{l}0.34 \\
\cdot\end{array}$ & $\begin{array}{l}124 \\
491 \\
407\end{array}$ & $\begin{array}{r}6 \\
19 \\
15\end{array}$ & $\begin{array}{l}0.088 \\
. \\
.\end{array}$ & $\begin{array}{l}110 \\
267 \\
149\end{array}$ & $\begin{array}{r}8 \\
14 \\
13\end{array}$ & $\begin{array}{l}0.20 \\
\cdot\end{array}$ & $\begin{array}{l}118 \\
333 \\
172\end{array}$ \\
\hline
\end{tabular}


Table 2. Continued.

\begin{tabular}{|c|c|c|c|c|c|c|c|c|c|c|c|c|}
\hline \multirow{3}{*}{ Factor } & \multicolumn{6}{|c|}{ Women } & \multicolumn{6}{|c|}{ Men } \\
\hline & \multicolumn{3}{|c|}{ Neck symptoms } & \multicolumn{3}{|c|}{ Hand-wrist symptoms } & \multicolumn{3}{|c|}{ Neck symptoms } & \multicolumn{3}{|c|}{ Hand-wrist symptoms } \\
\hline & $\%$ & P-value & $\mathrm{N}_{\text {total }} \mathrm{a}$ & $\%$ & P-value & $\mathrm{N}_{\text {total }}{ }^{\mathrm{a}}$ & $\%$ & P-value & $\mathrm{N}_{\text {lotal }}$ a & $\%$ & P-value & $\mathrm{N}_{\text {total }}{ }^{\mathrm{a}}$ \\
\hline \multicolumn{13}{|l|}{ Technical problems with computer } \\
\hline Daily or at least once a week & 29 & 0.096 & 280 & 22 & 0.39 & 451 & 19 & 0.12 & 140 & 14 & 0.30 & 170 \\
\hline At least once a month & 24 & & 194 & 22 & . & 295 & 17 & . & 172 & 13 & . & 211 \\
\hline Less than once a month & 22 & . & 176 & 19 & . & 273 & 13 & . & 213 & 11 & . & 241 \\
\hline \multicolumn{13}{|l|}{ Quality of technical support } \\
\hline Dissatisfactory & 27 & 0.38 & 154 & 20 & 0.94 & 231 & 16 & 0.62 & 99 & 18 & 0.029 & 120 \\
\hline Satisfactory & 25 &. & 405 & 22 &. & 642 & 16 & $\cdot$ & 334 & 12 & . & 395 \\
\hline Very satisfactory & 21 & . & 76 & 20 & . & 124 & 13 & . & 82 & 8 & . & 92 \\
\hline \multicolumn{13}{|c|}{ Space for arm support in front of keyboard } \\
\hline No & .. & . & & 24 & 0.27 & 243 & .. & . & . & 14 & 0.57 & 195 \\
\hline Yes & .. & . & & 21 & & 773 & .. & . & . & 12 & $\cdot$ & 410 \\
\hline \multicolumn{13}{|l|}{ Screen height } \\
\hline Top above eye level & 31 & 0.071 & 216 & .. & . & . & 15 & 0.76 & 143 & .. & . & . \\
\hline Top at or below eye level & 23 & 427 &.. & .. & . & . & 16 & & 380 & .. & . & . \\
\hline \multicolumn{13}{|l|}{ Disturbed by glare } \\
\hline Daily or several times a week & 32 & 0.056 & 115 & 24 & 0.043 & 187 & 15 & 0.88 & 82 & 18 & 0.024 & 101 \\
\hline Every once in a while & 25 & & 192 & 25 &. & 324 & 17 & .00 & 147 & 14 & & 179 \\
\hline No & 23 & . & 326 & 18 & . & 482 & 15 & . & 289 & 10 & . & 334 \\
\hline
\end{tabular}

a $\mathrm{N}_{\text {total }}=$ total number of nonsymptomatic respondents at baseline.

were risk factors for the development of symptoms among the women. Furthermore, persons with incident neck symptoms more often experienced technical problems with the computer and reported that their screen was placed with the top above eye level. Factors associated with the development of neck symptoms among the men in the univariate analyses were age and duration of employment (negative associations), high cognitive demands, sufficient training in software use, and good computer skills. Low influence at work, dissatisfactory quality of technical support, and disturbance by glare were associated with the development of handwrist symptoms among the men.

In the multivariate logistic regression models, two to three factors remained that contributed significantly to predicting the development of symptoms (tables 3 and 4). Previous symptoms remained in all the models as the most powerful predictor of developing more symptoms. In addition, influence at work remained in both models for the women (neck and hand-wrist models) and in the model for hand-wrist symptoms for the men as a significant predictor. Those with the highest risk of developing symptoms were women with low influence at work and men with medium low influence at work. Furthermore, a highly placed computer screen predicted neck symptoms among the women. Sensorial demands showed a U-shaped association with handwrist symptoms among the women. Among the men a short duration of employment in the same job and good computer skills predicted the development of neck
Table 3. Logistic regression models for neck and hand-wrist symptoms among the women. The P-value refers to the likelihood-ratio test for the models with all women.

\begin{tabular}{|c|c|c|c|c|c|}
\hline \multirow[b]{2}{*}{ Region } & \multicolumn{3}{|c|}{ All women } & \multicolumn{2}{|c|}{$\begin{array}{l}\text { Women with same } \\
\text { job in follow-up }\end{array}$} \\
\hline & OR & $95 \% \mathrm{Cl}$ & P-value & OR & $95 \% \mathrm{Cl}$ \\
\hline Neck & \multicolumn{3}{|c|}{$\mathrm{N}_{\text {total }}{ }^{a}=637$} & \multicolumn{2}{|c|}{$N_{\text {total }}{ }^{a}=516$} \\
\hline \multicolumn{6}{|c|}{ Previous neck or shoulder symptoms } \\
\hline No & 1.0 & & \multirow[t]{2}{*}{$<0.001$} & 1.0 & \\
\hline $1-7$ days & 2.8 & $1.9-4.1$ & & 2.9 & $1.9-4.5$ \\
\hline \multicolumn{6}{|c|}{ Screen height relative to eye level } \\
\hline Below & 1.0 & . & \multirow[t]{2}{*}{0.046} & 1.0 & \\
\hline Above & 1.5 & $1.0-2.2$ & & 1.4 & $0.9-2.2$ \\
\hline \multicolumn{6}{|l|}{ Influence at work } \\
\hline High & 1.0 & & \multirow[t]{4}{*}{0.013} & 1.0 & \\
\hline Medium high & 1.4 & $0.8-2.5$ & & 1.2 & $0.7-2.3$ \\
\hline Medium low & 2.1 & $1.2-3.6$ & & 2.0 & $1.1-3.6$ \\
\hline Low & 2.2 & $1.3-3.7$ & & 1.6 & $0.9-2.9$ \\
\hline Hand-wrist & \multicolumn{3}{|c|}{$N_{\text {total }}{ }^{a}=1016$} & \multicolumn{2}{|c|}{$\mathrm{N}_{\text {total }} \mathrm{a}^{\mathrm{a}}=800$} \\
\hline \multicolumn{6}{|c|}{ Previous hand, wrist or elbow symptoms } \\
\hline No & 1.0 & . & \multirow[t]{2}{*}{$<0.001$} & 1.0 & \\
\hline $1-7$ days & 3.3 & $1.6-2.4$ & & 3.5 & $2.5-5.1$ \\
\hline \multicolumn{6}{|c|}{ Sensorial demands } \\
\hline Low & 1.0 & . & 0.015 & 1.0 & . \\
\hline Medium low & 0.6 & $0.3-1.1$ & & 0.7 & $0.3-1.4$ \\
\hline Medium high & 0.8 & $0.5-1.3$ & . & 0.8 & $0.5-1.5$ \\
\hline High & 1.3 & $0.8-2.0$ & . & 1.3 & $0.8-2.1$ \\
\hline \multicolumn{6}{|l|}{ Influence at work } \\
\hline High & 1.0 & & $<0.001$ & 1.0 & \\
\hline Medium high & 1.5 & $0.9-2.5$ &. & 1.6 & $0.9-2.9$ \\
\hline Medium low & 2.3 & $1.5-3.8$ & . & 2.8 & $1.6-4.7$ \\
\hline Low & 2.4 & $1.5-3.8$ & . & 2.7 & $1.6-4.6$ \\
\hline
\end{tabular}

a $\mathrm{N}_{\text {total }}$ = total number of respondents included in the logistic regression model. 
Table 4. Logistic regression models for neck and hand-wrist symptoms among the men. The P-value refers to the likelihoodratio test for the model with all the men.

\begin{tabular}{|c|c|c|c|c|c|}
\hline \multirow[b]{2}{*}{ Region } & \multicolumn{3}{|c|}{ All men } & \multicolumn{2}{|c|}{$\begin{array}{l}\text { Men with same } \\
\text { job in follow-up }\end{array}$} \\
\hline & OR & $95 \% \mathrm{Cl}$ & P-value & OR & $95 \% \mathrm{Cl}$ \\
\hline Neck & \multicolumn{3}{|c|}{$\mathrm{N}_{\text {total }}{ }^{a}=517$} & \multicolumn{2}{|c|}{$N_{\text {total }}{ }^{a}=399$} \\
\hline \multicolumn{6}{|c|}{ Previous neck or shoulder symptoms } \\
\hline No & 1.0 & & \multirow{2}{*}{$<0.001$} & 1.0 & \multirow{2}{*}{$2.6-8.5$} \\
\hline $1-7$ days & 3.8 & $2.3-6.5$ & & 4.7 & \\
\hline \multicolumn{6}{|c|}{ Duration of employment in same job } \\
\hline$>3$ years & 1.0 & . & \multirow[t]{3}{*}{0.091} & 1.0 & . \\
\hline $1-3$ years & 1.4 & $0.8-2.5$ & & 1.8 & $1.0-3.4$ \\
\hline$<1$ year & 2.1 & $1.1-3.9$ & & 2.1 & $1.0-4.5$ \\
\hline \multicolumn{6}{|l|}{ Computer skills } \\
\hline Very good & 1.0 & - & \multirow[t]{3}{*}{0.005} & 1.0 & . \\
\hline Good & 1.2 & $0.7-2.1$ & & 1.3 & $0.7-2.5$ \\
\hline Less than good & 0.4 & $0.1-0.9$ & & 0.4 & $0.2-1.1$ \\
\hline Hand-wrist & \multicolumn{2}{|c|}{$\mathrm{N}_{\text {total }}{ }^{\mathrm{a}}=621$} & \multicolumn{2}{|l|}{$\mathrm{N}_{\text {otal }}{ }^{a}=479$} & \\
\hline \multicolumn{6}{|c|}{ Previous hand, wrist or elbow symptoms } \\
\hline No & 1.0 & . & \multirow[t]{2}{*}{$<0.001$} & 1.0 & . \\
\hline $1-7$ days & 3.3 & $2.0-5.3$ & & 3.3 & $1.9-5.7$ \\
\hline \multicolumn{6}{|l|}{ Influence at work } \\
\hline High & 1.0 & . & \multirow{4}{*}{0.021} & 1.0 & . \\
\hline Medium high & 2.2 & $1.2-4.0$ & & 2.0 & $1.0-3$ \\
\hline Medium low & 2.5 & $1.3-4.8$ & & 2.4 & $1.2-5.1$ \\
\hline Low & 1.6 & $0.6-4.0$ & & 1.1 & $0.4-3.6$ \\
\hline
\end{tabular}

${ }^{a} \mathrm{~N}_{\text {total }}=$ total number of respondents included in the logistic regression model.

Table 5. Odds ratios (OR) and 95\% confidence intervals (95\% C) for symptoms in relation to the duration of computer use at work (as a fraction of the worktime) and the duration of mouse use at work (as a fraction of the worktime). The odds ratios were controlled for gender (model for duration of computer use), age and other factors that were associated with the development of symptoms (see tables 3 and 4). Only subjects who worked fulltime (32-41 hours/week) and remained in the same job during the follow-up period were included. The models with mouse use were further restricted to the women who used a computer almost continually. The P-values refer to the likelihood-ratio test.

\begin{tabular}{|c|c|c|c|c|}
\hline Region & OR & $95 \% \mathrm{Cl}$ & P-value & $\mathrm{N}$ \\
\hline \multicolumn{5}{|c|}{ Neck } \\
\hline \multicolumn{5}{|c|}{ Worktime at computer } \\
\hline $0-25 \%$ & 1.0 & . & 0.541 & 645 \\
\hline $50 \%$ & 1.5 & $0.7-3.1$ & $\cdot$ & $\cdot$ \\
\hline $75 \%$ & 1.3 & $0.6-2.7$ & . & . \\
\hline $100 \%$ & 1.6 & $0.8-3.3$ & . & . \\
\hline \multicolumn{5}{|c|}{ Worktime using mouse } \\
\hline Seldom & 1.3 & $0.4-4.3$ & 0.718 & 203 \\
\hline $25 \%$ & 1.0 & & . & . \\
\hline $50-100 \%$ & 1.7 & $0.5-5.7$ & . & . \\
\hline \multicolumn{5}{|l|}{ Hand-wrist } \\
\hline \multicolumn{5}{|c|}{ Worktime at computer } \\
\hline $0-25 \%$ & 1.5 & $0.7-3.4$ & 0.062 & 916 \\
\hline $50 \%$ & 1.0 & & . & . \\
\hline $75 \%$ & 2.0 & $1.1-3.9$ & . & . \\
\hline $100 \%$ & 2.3 & $1.2-4.3$ & . & . \\
\hline \multicolumn{5}{|c|}{ Work time using mouse } \\
\hline Seldom & 4.0 & $1.1-14.4$ & 0.050 & 331 \\
\hline $25 \%$ & 1.0 & . & . & . \\
\hline $50-100 \%$ & 4.0 & $1.0-15.5$ & . & . \\
\hline
\end{tabular}

symptoms in the multivariate analyses. When the final logistic regression models were recalculated with only the respondents who held the same job in the follow-up period, the odds ratios were similar to those of the models in which also the respondents who had changed jobs were included (tables 3 and 4). The only exceptions were the odds ratios for low influence at work, which were reduced when the respondents who had changed jobs were excluded. In addition, when the duration of computer use (as a fraction of worktime) was included in the models, the odds ratios for the other factors were similar to those of the models without this factor (results not shown).

The duration of computer use was not significantly associated with neck symptoms (table 5). However, duration of computer use predicted hand-wrist symptoms (table 5). Those with computer use for about onehalf of their worktime showed the lowest risk, and in comparison those with almost continual computer use showed an odds ratio of 2.3 for hand-wrist symptoms.

The association between mouse use and neck symptoms was analyzed for women with almost continual computer use (table 5). No significant association was found. However, mouse use was associated with handwrist symptoms. Thus mouse use for at least half of the worktime as compared with mouse use for one-fourth of the worktime showed an odds ratio of about 4. Intensive computer use without mouse use also showed an odds ratio of about 4 , in comparison with mouse use for one-fourth of the worktime.

\section{Discussion}

\section{Response rates}

The response rate was $77 \%$ in the follow-up, and there could have been selection bias, especially if people without musculoskeletal symptoms were more likely to drop out at follow-up than symptomatic ones. However, the prevalence of symptoms at baseline was similar among those who remained in the study and those who responded at baseline. This finding indicates that there was no selection bias due to musculoskeletal health status. In addition, no sign of selection bias regarding gender was observed. Still, almost half of the subjects who received a questionnaire at baseline did not respond either at baseline or at follow-up, and therefore some uncertainty remains about the size of the reported effects.

\section{Analysis strategy}

Durations of computer and mouse use were not included in the first logistic regression models, where all other 
factors were potential candidates for inclusion (tables 3 and 4). One purpose of these analyses was to identify general risk factors among computer users, as they have seldom been verified in follow-up studies. The time span between the registration of potential risk factors and outcome (symptoms $>7$ days within the last year) increased the possibility that the identification of risk factors was due to cause-response relationships when a comparison is made to previous cross-sectional studies. The final models were repeated including only subjects who had not changed jobs in the follow-up period. In the follow-up, questions on most of the exposure factors were not repeated, and therefore the confirmation that the subject had not changed jobs was the best way of minimizing errors associated with a change in exposure during the follow-up period. However, this approach affected the final models only marginally. There was no tendency towards a higher frequency of respondents with symptoms or intense computer use among those who had changed jobs; this finding indicates that the computer users mainly changed jobs for other reasons. In addition, the respondents with symptoms at baseline did not show a greater tendency towards a reduced duration of computer use at follow-up than those without symptoms (data not shown).

Another purpose of the first analysis was to control for identified risk factors in the analyses of relationships between duration of computer use and symptoms in order to estimate the effect of computer use per se. Furthermore, the question on duration of computer use was answered in relation to the total workhours of each subject. Thus the analyses of the effect of duration of computer use were also limited to subjects who worked fulltime (32-41 hours/week) and had not changed jobs so that exposures would be as comparable as possible between the subjects and time periods. Therefore, the effects of duration of computer use were analyzed in separate logistic regression models. The effects of duration of mouse use were also analyzed separately, as the analyses were restricted to subjects with almost continual computer use in addition to the same restrictions used in the former analyses of the effects of duration of computer use.

\section{Computer and mouse use}

The duration of computer use at work predicted handwrist symptoms but not neck symptoms. The risk of developing symptoms was increased in work with the computer for three-fourths or more of the worktime as compared with one-half of the worktime or less. The odds ratios were similar before and after control for other factors that affected the development of symptoms. The importance of the duration of computer work for the development of symptoms has previously been indicated by several cross-sectional studies $(1,4,6,7$, 10). In the follow-up study by Marcus et al (12), the risk of developing hand-arm symptoms increased with hours of keying among newly hired computer users with more than 15 hours of computer use per week. The risk increased more than twofold with an increase of 20 hours of keying, whereas the risk of developing neck-shoulder symptoms did not increase significantly with hours of keying. This finding is probably consistent with my present findings, even though hours of keying was not studied independently. In the present study the analyses were restricted to those working "normal" hours (ie, 32-41 hours/week). Thus it is not known whether longer workhours increase the risk of developing hand-wrist symptoms further, as computer users who typically work longer hours may have other types of jobs, and other factors may influence the development of symptoms more or less than for the "normal" computer user.

Using the computer mouse for more than one-half of the worktime also increased the risk of developing hand-wrist symptoms for women when compared with using the mouse for about a quarter of the worktime. This finding is consistent with the results of previous cross-sectional studies $(6,8)$. Typical worktasks requiring intensive mouse use would be graphic design and multimedia editing. However, intense computer use without a mouse also increased the risk of developing hand-wrist symptoms, probably due to the performance of rather repetitive worktasks with intense keyboard use, such as call-center work or data entry.

\section{Gender and age}

Women were more likely to develop symptoms than men. In most cross-sectional studies of computer users, the prevalence of musculoskeletal symptoms has also been about twice as high for women as for men $(3,10)$. In a recent follow-up study, the effect of gender was slightly smaller, as women showed a 1.6-1.7 times higher risk of developing symptoms than men (11). However, the risk of developing clinically assessed hand-arm disorders was 2.4 times higher for women than for men in the same study. Part of the explanation may be that women often perform other worktasks or jobs than men, such as call-center work. Furthermore, other studies have shown that identical worktasks at the computer may be performed with more strenuous postures by women than by men $(16,17)$, probably due to anthropometric differences.

Age did not remain a predictor in the multivariate models. However, having performed the same worktasks for less than a year prior to the baseline survey was associated with a higher risk of developing neck symptoms among the men than having performed the same tasks for more than 3 years. This finding indicated that 
employees who have little experience in performing their worktask have a higher risk of developing musculoskeletal problems than their more experienced colleagues. Differences in job content or healthy worker selection may partly explain this result. This finding is also consistent with the findings of Gerr et al (11), who reported a considerable increase in the occurrence of musculoskeletal symptoms during the first year after being hired in a new job.

\section{Previous symptoms}

The strongest predictor of new musculoskeletal symptoms for more than 7 days within the last year was previous symptoms (ie, symptoms for $<7$ days in the year prior to the baseline survey). Westgaard et al (18) used previous symptoms as an indicator of individual sensitivity to the development of symptoms; however, previous symptoms were defined differently than in the present paper. If some office workers almost never experience symptoms, "previous symptoms" may also here indicate a higher sensitivity among some persons. However, the high incidence relative to the prevalence also indicated that the nature of musculoskeletal symptoms is dynamic even from one year to another. Thus most respondents with symptoms may experience infrequent symptoms during some years and more frequent symptoms during others. An additional explanation for the relatively high risk associated with previous symptoms could be misclassification of the duration of symptoms. Thus respondents with symptoms for 1-7 days within the last year at baseline were more likely to report $>7$ days of symptoms at follow-up than respondents with no symptoms at baseline simply due to errors of recall. Thus no conclusive interpretation of the risk associated with this factor can be made.

\section{Psychosocial factors}

Low influence at work was a risk factor for the development of neck symptoms. Among the women low or medium low influence at work was also a risk factor for hand-wrist symptoms. Questions on influence at work primarily referred to the possibilities for the employees to organize their own work. The higher risk of developing symptoms for those with low influence could be due to the lack of possibilities to take breaks if needed and to plan the sequence of worktasks in a way that was most comfortable for the individual employee.

Women with high sensorial demands were more likely to develop hand-wrist symptoms than women with medium low demands. This finding emphasized the importance of specific job demands and job control as work environment factors that predict the health of computer users. Faucett $\&$ Rempel (5) also reported that low job control was associated with an increased prevalence of symptoms. In my study several dimensions of job demands were examined, as it is important to identify the specific dimension(s) that predict the development of symptoms. The dimensions (indices) and the questions contained in each index were developed by Kristensen et al (14), who used these questions in psychosocial surveys of people from the general workforce. When compared with their data, respondents who used computers for a large part of their worktasks in the present study were exposed to high sensorial demands when compared with those with many other job types. Furthermore, high sensorial demands provide an especially relevant focus during computer work, as a poorly designed user interface may increase the demands for vision and precision more than necessary. Such demands may increase the duration of static work postures and thereby the risk of developing musculoskeletal symptoms.

A surprising finding in the present study was the lower risk of developing neck symptoms among the men with less than good computer skills as compared with those with good skills. Probably low skills do not have a preventive effect, but other factors that could explain this association were not characterized well enough to exclude computer skills from the final model of the multivariate analyses. The association was not found for the women.

\section{Workplace design}

It is generally agreed that workplace design can affect the risk of developing musculoskeletal symptoms (2). A recent longitudinal study used workplace observations to identify postural factors that were risk factors for the development of symptoms among computer users in relation to workplace design (12). However, the study also reported a lack of association between several postural factors and the development of symptoms. Except for factors easily assessed by respondents, it is difficult to study or control factors related to workplace design in a questionnaire study. In the present study a screen height with the top of the screen above eye level was found to predict neck symptoms among women. This finding is consistent with generally accepted recommendations for optimal workplace design. However, the same phenomenon was not found for the men. The risk of developing symptoms when workplace design is nonoptimal may be due to work in postures that are constrained and involve more static muscle loads than otherwise needed.

\section{Concluding remarks}

To prevent hand-wrist symptoms among computer users with normal workhours (32-41 hours/week) computer 
use should be limited to less than three-fourths of the worktime. Worktasks requiring mouse use for at least one-half of the worktime were found to be associated with an increased risk of hand-wrist symptoms. Furthermore, low and medium low influence at work predicted both neck symptoms for women and hand-wrist symptoms for women and men. Associations between symptoms and sensorial demands, good computer skills, low duration of employment in the same job, and screen height were not consistently observed for both women and men and should therefore be interpreted with caution.

\section{Acknowledgments}

The BIT study was initiated as part of research collaboration involving both epidemiologic and laboratory studies focusing on the musculoskeletal problems of computer users. Therefore, I thank K Hansen, AK Blangsted, L Finsen, K Søgaard, CU Ryholt, H Burr, E Villadsen, and H Christensen for their efforts during the course of the BIT study.

\section{References}

1. Jensen C, Finsen L, Søgaard K, Christensen H. Musculoskeletal symptoms and duration of computer and mouse use. Int $\mathbf{J}$ Ind Ergron 2002;30:265-75.

2. Punnett L, Bergqvist U. Visual display unit work and upper extremity musculoskeletal disorders: a review of epidemiological findings. Stockholm: National Institute for Working Life; 1997. p 1-161. Ergonomic expert committee document no 1.

3. Jensen C, Ryholt CU, Burr H, Villadsen E, Christensen H. Work-related psychosocial, physical and individual factors associated with musculoskeletal symptoms in computer users. Work Stress 2002;16(2):107-20.

4. Bernard B, Sauter S, Fine L, Petersen M, Hales T. Job task and psychosocial risk factors for work-related musculoskeletal disorders among newspaper employees. Scand J Work Environ Health 1994;20:417-26.

5. Faucett J, Rempel D. VDT-related musculoskeletal symptoms: interactions between work posture and psychosocial work factors. Am J Ind Med 1994;26:597-612.

6. Karlqvist L, Hagberg M, Köster M, Wenemark M, Ånell R. Musculoskeletal symptoms among computerassisted design (CAD) operators and evaluation of a self-assessment questionnaire. Int J Occup Environ Health 1996;2:185-94.

7. Fogleman M, Lewis RJ. Factors associated with self-reported musculoskeletal discomfort in video display terminal (VDT) users. Int J Ind Ergon 2002;29:311-8.

8. Jensen C, Borg V, Finsen L, Hansen K, Juul-Kristensen B, Christensen H. Job demands, muscle activity and musculoskeletal symptoms in relation to work with the computer mouse. Scand J Work Environ Health 1998;24:418-24.

9. Bergqvist U, Wolgast E, Nilsson B, Voss M. Musculoskeletal disorders among visual display terminal workers: individual, ergonomic, and work organizational factors. Ergonomics 1995;38:763-76.

10. Polanyi MFD, Cole DC, Beaton DE, Chung J, Wells R, Abdolell M, et al. Upper limb work-related musculoskeletal disorders among newspaper employees: cross-sectional survey results. Am J Ind Med 1997;32:620-8.

11. Gerr F, Marcus M, Ensor C, Kleinbaum D, Cohen S, Edwards A, et al. A prospective study of computer users: I. Study design and incidence of musculoskeletal symptoms and disorders. Am J Ind Med 2002;41:221-35.

12. Marcus M, Gerr F, Monteilh C, Ortiz DJ, Gentry E, Cohen S, et al. A prospective study of computer users: II. Postural risk factors for musculoskeletal symptoms and disorders. Am J Ind Med 2002;41:236-49.

13. Bergqvist U, Knave B, Wibom R, Voss M. A longitudinal study of VDT work and health. Int J Hum Comput Interact 1992;4:197-219.

14. Kristensen TS, Borg V, Hannerz H. Socioeconomic status and psychosocial work environment: result from a national Danish study. Scand J Public Health 2002;30:41-8.

15. Kuorinka I, Jonsson B, Kilbom ^̊, Vinterberg H, BieringSørensen F, Andersson G, et al. Standardised Nordic questionnaires for the analysis of musculoskeletal symptoms. Appl Ergon 1987;18:233-7.

16. Karlqvist L, Bernmark E, Ekenvall L, Hagberg M, Isaksson A, Rostö T. Computer mouse and trackball operation: similarities and differences in posture, muscular load and perceived exertion. Int J Ind Ergon 1999;23:157-69.

17. Wahlström J, Svensson J, Hagberg M, Johnson PW. Differences between work methods and gender in computer mouse use. Scand J Work Environ Health 2000;25:390-7.

18 Westgaard RH, Jensen C, Hansen K. Individual and workrelated risk factors associated with symptoms of musculoskeletal complaints. Int Arch Occup Environ Health 1993;64:40513.

Received for publication: 21 August 2002 